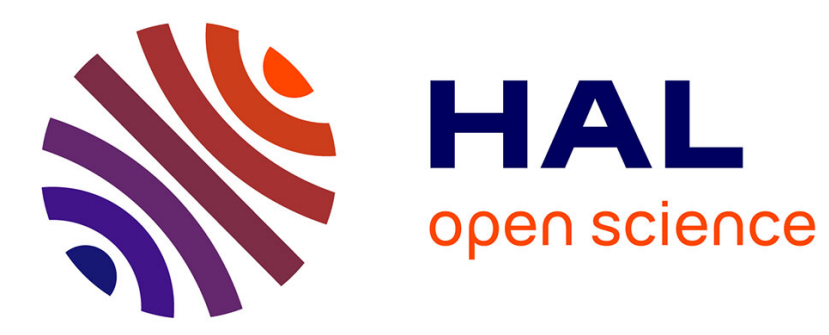

\title{
Channel Estimation for orthogonal STBC MC-CDMA systems over realistic MIMO channels
}

Stephan Sand, Fabrice Portier, Jean-François Hélard

\section{To cite this version:}

Stephan Sand, Fabrice Portier, Jean-François Hélard. Channel Estimation for orthogonal STBC MCCDMA systems over realistic MIMO channels. 2006. hal-00082812

\section{HAL Id: hal-00082812 \\ https://hal.science/hal-00082812}

Submitted on $20 \mathrm{Jul} 2006$

HAL is a multi-disciplinary open access archive for the deposit and dissemination of scientific research documents, whether they are published or not. The documents may come from teaching and research institutions in France or abroad, or from public or private research centers.
L'archive ouverte pluridisciplinaire HAL, est destinée au dépôt et à la diffusion de documents scientifiques de niveau recherche, publiés ou non, émanant des établissements d'enseignement et de recherche français ou étrangers, des laboratoires publics ou privés. 


\section{Channel Estimation for Orthogonal STBC MC-CDMA Systems over Realistic MIMO Channels}

\author{
Stephan Sand \\ German Aerospace Center \\ Institute of Communications and Navigation \\ Oberpfaffenhofen, 82234 Wessling, Germany \\ Email: stephan.sand@dlr.de
}

\author{
Fabrice Portier, Jean-François Hélard \\ Institute of Electronics and Telecommunications in Rennes \\ Institut National des Sciences Appliquées \\ 20 Ave. des Buttes de Coësmes, 35043 Rennes Cedex, France \\ Email: fabrice.portier@insa-rennes.fr
}

\begin{abstract}
This paper investigates a downlink multiple-input multiple-output (MIMO) multi-carrier code division multiple access (MC-CDMA) system with pilot aided channel estimation (PACE) and iterative channel estimation (ICE) in the receiver. Due to the superposition of different users' spread data signals, zero-valued chips can occur after spreading, which can cause noise enhancement when using data estimates as reference signals in ICE. Hence, we propose a MIMO channel estimation method to overcome the above problem and avoid noise enhancement in ICE. Simulations results for PACE and ICE were computed for a realistic outdoor MIMO channel model. The results show that frame error rates of ICE are smaller than the ones of PACE.
\end{abstract}

\section{INTRODUCTION}

Orthogonal frequency division multiplexing (OFDM) [1] is a suitable technique for broadband transmission over multipath fading radio channels achieving high data rates. In addition to the coherent OFDM modulation, spreading in frequency or time direction is introduced for multi-carrier code division multiple access (MC-CDMA). MC-CDMA is a promising candidate for the downlink of future mobile communication systems and has been implemented in experimental systems by NTT DoCoMo [2] and the European IST projects MATRICE [3] and 4MORE [4]. High data rate MC-CDMA systems can additionally employ multiple-input and multiple-output (MIMO) techniques, e.g., the Alamouti space-time block code (STBC) [5].

Coherent STBC OFDM systems require channel state information (CSI) at the receiver. Thus, pilot symbols are often periodically inserted into the transmitted signal to support channel estimation (CE). CE is performed by interpolating the time-frequency pilot grid and exploiting the correlations of the received OFDM signal, which are introduced by the time- and frequency-selective radio channel. Exploiting these correlations, the pilot aided channel estimation (PACE) is performed by cascading two one-dimensional (1-D) finiteimpulse response filters whose coefficients are based on the minimum mean square error (MMSE) criterion [1].

To further improve PACE, [6]-[8] use previously decided data symbols as reference in iterative channel estimation (ICE). In [6], the authors propose an ICE algorithm for OFDM that feeds back information from the output of the channel decoder to the estimation stage to reduce decision feed-back errors. Since the CE gets additional information from the estimated data symbols, ICE achieves a further reduction of the bit error rate (BER).

Previously, we proposed several MIMO MC-CDMA systems and studied the effect of CE errors on these 4-th Generation $(4 \mathrm{G})$ schemes [9]. In [8], we proposed SISO ICE methods, in particular the modified LS ICE method to solve the problem of zero-valued chips after spreading in ICE. In this paper, we extend the idea of ICE to a MIMO MC-CDMA system with Walsh-Hadamard (WH) spreading codes. At the transmitter, we employ orthogonal STBCs, such as the Alamouti scheme. We compare the performance of PACE and ICE for a downlink MIMO MC-CDMA system with two transmit and two receive antennas. Simulation results are obtained for a realistic outdoor MIMO channel based on the 3GPP/SCM MIMO channel model [10].

\section{SYSTEM MODEL}

Figure 1 represents the block diagram of the downlink MIMO MC-CDMA system with ICE. At the transmitter, a binary signal of a single user out of $K$ active users is encoded by a channel coder and interleaved by a random bit interleaver. The bits $b_{k}$ (Figure 1(b)) are modulated and serial-to-parallel converted to $M$ data symbols per user in an OFDM symbol. After spreading with a WH sequence of length $L(L \geq K)$, the spread signals are combined and serial-to-parallel converted to form the data symbol vector $\underline{S}_{l}=\left[S_{1, l}, \ldots, S_{N_{\mathrm{c}}, l}\right]^{T} \quad(\{l\} \in$ $\mathcal{D})$. Here, $N_{\mathrm{c}}$ denotes the number of subcarriers per OFDM symbol, $l$ the OFDM symbol number, and $\mathcal{D}$ the set of data symbol positions in a frame. Next, $\underline{S}_{l}$ (Figure 1(a)) is spacetime coded and each $\underline{S}_{l}^{m}\left(\{l\} \in \mathcal{D}^{m}, m=1, \ldots, N_{\mathrm{TX}}\right)$ is multiplexed together with pilot symbols $\underline{S}_{l^{\prime}}^{m},\left\{l^{\prime}\right\} \in \mathcal{P}$, $m=1, \ldots, N_{\mathrm{TX}} \cdot \mathcal{P}$ denotes the set of all pilot symbol positions in a frame. Here, $N_{\mathrm{TX}}=M \cdot L$ subsequent full OFDM pilot symbols are inserted at the beginning, in the middle and at the end of each frame (Figure 1(c)). For each transmit antenna, a unique WH spreading code is applied to the pilot symbols in time direction. If the time variation of the channel is small enough over the consecutively spread pilots, the pilot symbols for each transmit antenna can be despread at the receiver to obtain localized estimates (LEs) of the CSI. Here, we assume that the pilot and data sets are disjoint, i.e., $\mathcal{P} \cap \mathcal{D}=\emptyset$. The resulting $N_{\mathrm{TX}}$ frames with 
$N_{\mathrm{c}}$ subcarriers and $N_{\mathrm{s}}$ OFDM symbols are OFDM modulated and cyclically extended by the guard interval (GI) before they are transmitted over a time-variant MIMO multipath channel, which adds white Gaussian noise.

The received symbols are shortened by the GI and OFDM demodulated for each of the $N_{\mathrm{RX}}$ receive antennas. Then, the received pilot symbols $\underline{\hat{R}}_{l^{\prime}}^{p}\left(\left\{l^{\prime}\right\} \in \mathcal{P}, p=1, \ldots, N_{\mathrm{RX}}\right)$ are separated from the received data symbols $\underline{R}_{l}^{p}(\{l\} \in \mathcal{D})$, and fed into the CE. In the initial ICE iteration $(i=0)$, the CE only uses pilot symbols to estimate the CSI.

After demultiplexing and space-time decoding the received data symbols $\underline{R}_{l}^{p}(\{l\} \in \mathcal{D})$, either one multi-user detector (MUD) or $K$ single-user detector (SUD) blocks return softcoded bits $\hat{b}_{k}^{(i)}$ of all users [8]. Subsequently, the bits are used to reconstruct the transmit signal $\underline{\check{S}}_{l}^{(i), m}, m=1, \ldots, N_{\mathrm{TX}}$, in ICE (Figure 1(a)).

In the $i$ th iteration of ICE $(i>0)$, the CE exploits the received pilot symbols $\underline{\hat{R}}_{l^{\prime}}^{p}\left(\left\{l^{\prime}\right\} \in \mathcal{P}\right)$, the received data symbols $\underline{R}_{l}^{p}(\{l\} \in \mathcal{D})$, and the reconstructed transmit signal $\underline{S}_{l}^{(i), m}\left(\{l\} \in \mathcal{D}, m=1, \ldots, N_{\mathrm{TX}}\right)$ to improve the accuracy of the CSI estimates. The newly obtained CSI estimates are fed back to the space-time decoder and the MUD/SUD block to improve the estimates of the transmitted bits.

\section{Pilot Aided Channel Estimation (PACE)}

Since the pilot symbols for different antennas are transmitted on the same positions, we first need to compute LEs of the CSI for each pair of transmit and receive antenna. For notational convenience, we drop the subcarrier and symbol index. $N_{\mathrm{TX}}$ consecutive pilot symbols in time direction at each transmit antenna are then given by

$$
\left[\dot{S}_{1}^{m}, \ldots, \dot{S}_{N_{\mathrm{TX}}}^{m}\right]^{T}=\underline{w}_{m} \cdot \dot{S},
$$

where $\underline{w}_{m}$ is the $m$-th column of the $N_{\mathrm{TX}} \times N_{\mathrm{TX}} \mathrm{WH}$ spreading matrix [1] and $\dot{S}$ is a pilot symbol. Note, $\underline{w}_{m}^{T} \underline{w}_{p}$ is one only for $m=p$ and otherwise zero. The averaged LE $\check{H}^{m, p}$ of the channel transfer function between transmit antenna $m$ and receive antenna $p$ of the pilot symbol block is obtained by despreading the $N_{\mathrm{TX}}$ consecutively received pilots $\dot{R}_{1}^{p}, \ldots, \hat{R}_{N_{\mathrm{TX}}}^{p}$ with the WH spreading vector $\underline{w}_{m}$, i.e.,

$$
\check{H}^{m, p}=\frac{1}{\dot{S}} \underline{w}_{m}^{T}\left[\dot{R}_{1}^{p}, \ldots, \dot{R}_{N_{\mathrm{TX}}}^{p}\right]^{T} .
$$

Assuming that the channel is approximately constant for the length of $\underline{w}_{m}$, we obtain

$$
\check{H}^{m, p}=H^{m, p}+\frac{1}{\bar{S}^{\prime}} \underline{w}_{m}^{T} Z^{p},
$$

where $Z^{p}$ denotes an equivalent additive white Gaussian noise component at the $p$ th receive antenna.

The final estimates of the channel transfer function are obtained from the LEs $\check{H}_{n^{\prime}, l^{\prime}}^{m, p y}$ two-dimensional (2-D) filtering:

$$
\hat{H}_{n, l}^{m, p}=\sum_{\left\{n^{\prime}, l^{\prime}\right\} \in \mathcal{T}_{n, l}} \omega_{n^{\prime}, l^{\prime}} \check{H}_{n^{\prime}, l^{\prime}}^{m, p}, \quad \mathcal{T}_{n, l} \subseteq \mathcal{P}, \forall\{n, l\} \in \mathcal{D},
$$

where $\omega_{n^{\prime}, l^{\prime}}$ is the 2-D filter impulse response. The subset $\mathcal{T}_{n, l} \subseteq \mathcal{P}$ is the set of LEs $\check{H}_{n^{\prime}, l^{\prime}}^{m, p}$ used to obtain $\hat{H}_{n, l}^{m, p}$.
The optimum solution of (4) in the MSE sense is the 2D Wiener filter [1]. Assuming the delay and Doppler power spectral densities (PSDs) to be statistically independent, the 2-D filter can be replaced by two cascaded 1-D filters, one for filtering in frequency and one for filtering in time direction. Since in practice the channel statistics are not perfectly known at the receiver, the CE filters are designed robust using for example a uniform Doppler PSD ranging from $-f_{\mathrm{D}_{\text {filter }}}$ to $f_{\mathrm{D}_{\text {filter }}}$. Note, the maximum Doppler frequency $f_{D_{\max }}$ of the channel can be different from the maximum Doppler frequency $f_{\mathrm{D}_{\text {filter }}}$ of the CE filters.

\section{Iterative Channel Estimation (ICE)}

In the following, we investigated ICE in detail for a MIMO MC-CDMA system. [8] demonstrates that due to the superposition of WH spread data signals zero-valued chips can occur in the transmit signal. For instance, a zero-valued chip occurs with $2 \%$ probability for a 4-QAM symbol alphabet and a WH spreading code of length 32 . Consequently, we need to take special care when computing the LEs at the data symbol positions. Otherwise, zero-valued or small amplitude chips can cause noise enhancement in ICE.

When employing orthogonal space time block codes (STBCs), e.g., the Alamouti scheme, we can exploit the orthogonality to compute $\check{H}^{(i), m, p}$. This is in contrast to [7], where the authors use interference cancellation to compute the LEs from different transmit antennas. Exploiting the orthogonality in the case of the Alamouti scheme [5], (2) becomes for the data estimates

$$
\left[\begin{array}{c}
\check{H}^{(i), 1, p} \\
\check{H}^{(i), 2, p}
\end{array}\right]=\frac{\sqrt{2}}{\left(\mathcal{S}^{(i)}\right)^{2}}\left[\begin{array}{cc}
\check{S}_{1}^{(i)} & \check{S}_{2}^{(i)} \\
-\left(\check{S}_{2}^{(i)}\right)^{*} & \left(\check{S}_{1}^{(i)}\right)^{*}
\end{array}\right]^{H} \cdot\left[\begin{array}{c}
R_{1}^{p} \\
R_{2}^{P}
\end{array}\right],
$$

where ${ }^{H}$ denotes the hermitian transpose of a matrix and $\left(\mathcal{S}^{(i)}\right)^{2}=\left|\check{S}_{1}^{(i)}\right|^{2}+\left|\check{S}_{2}^{(i)}\right|^{2}$. Note that due to the WH spreading $\check{S}_{1}^{(i)}$ and $\check{S}_{2}^{(i)}$ can be both zero. Therefore, we propose to use the following estimates for $\breve{H}^{(i), m, p}$

$$
\left[\begin{array}{l}
\check{H}^{(i), 1, p} \\
\check{H}^{(i), 2, p}
\end{array}\right]=\left\{\begin{array}{cc}
\frac{\sqrt{2}}{\left(\mathcal{S}^{(i)}\right)^{2}}\left[\begin{array}{cc}
\check{S}_{1}^{(i)} & \check{S}_{2}^{(i)} \\
-\left(\check{S}_{2}^{(i)}\right)^{*} & \left(\check{S}_{1}^{(i)}\right)^{*}
\end{array}\right]^{H} \cdot\left[\begin{array}{l}
R_{1}^{p} \\
R_{2}^{p}
\end{array}\right], \\
\text { if }\left(\mathcal{S}^{(i)}\right)^{2}>\rho_{\mathrm{th}}, \\
{\left[\begin{array}{l}
0 \\
0
\end{array}\right],} & \text { if }\left(\mathcal{S}^{(i)}\right)^{2} \leq \rho_{\mathrm{th}} .
\end{array}\right.
$$

With (6), the LEs will not cause noise enhancement and degrade the channel estimates in any iteration of ICE.

After reconstructing the transmitted signal from the estimated information bits, the estimated data symbols and the transmitted pilot symbols form the set $\mathcal{P}_{\text {ICE }}=\mathcal{P} \cup \mathcal{D}$ of reference symbols known at the receiver. The set $\mathcal{P}_{\text {ICE }}$ defines the complete frame of pilot and data symbols. For ICE with one or more iterations $(i>0)$, the following steps have to be executed in each iteration:

1) Reconstruct the transmit signal $\check{S}_{l}^{(i), m} \forall\{l\} \in \mathcal{D}$ and $m=1, \ldots, N_{\mathrm{TX}}$ from the estimated information bits. 


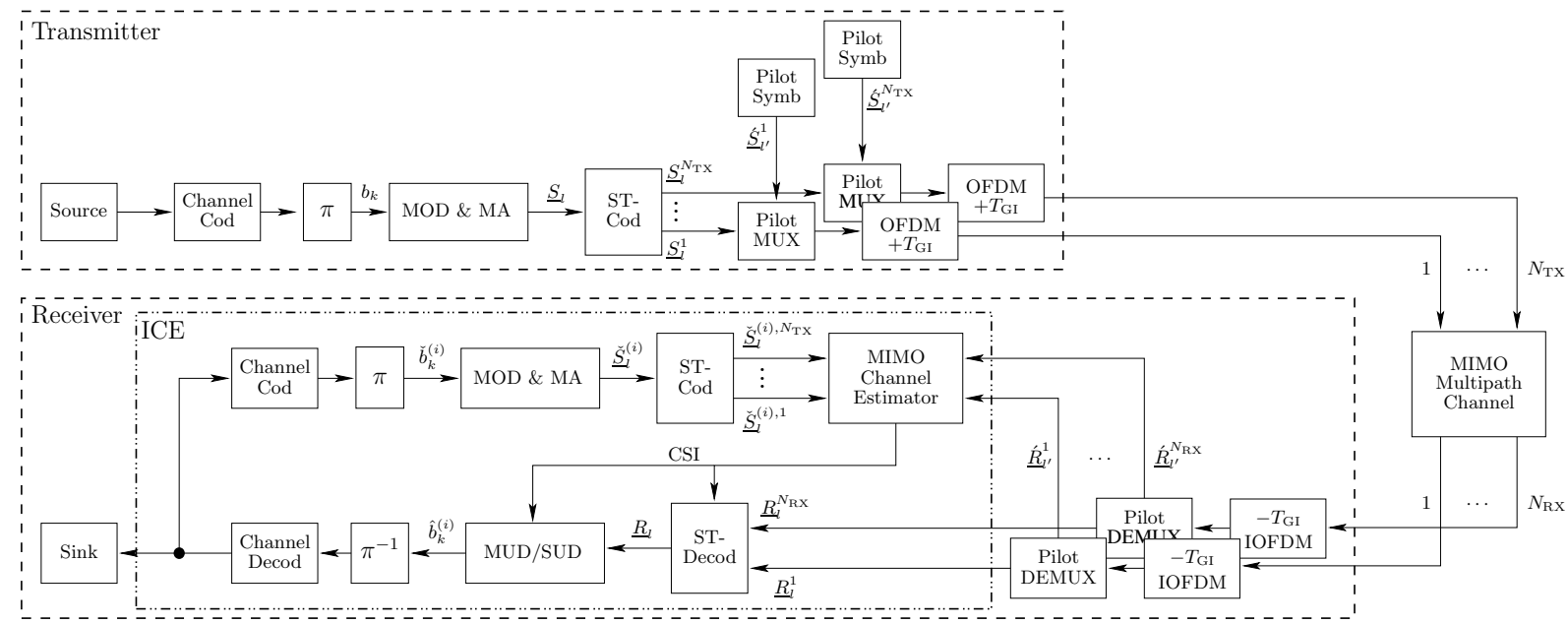

(a) MIMO MC-CDMA: transmitter and receiver with ICE

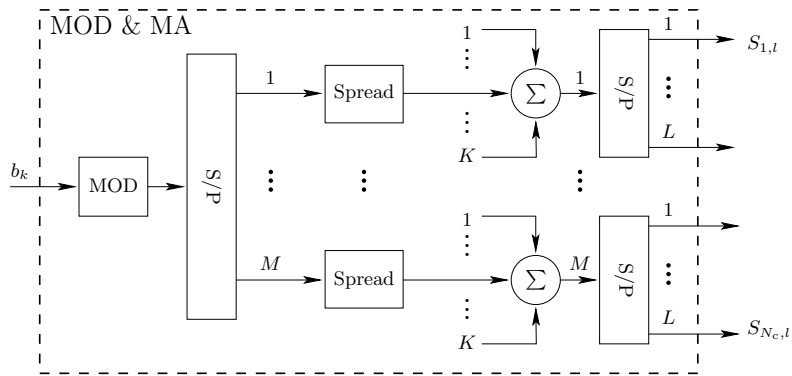

(b) Modulation and multiple access: MOD \& MA

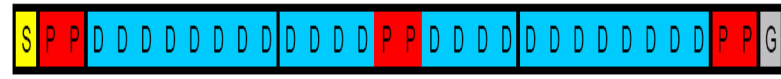

(c) Downlink frame: $\mathrm{S}=$ synchronization, $\mathrm{P}=$ pilot, $\mathrm{G}=$ guard, and $\mathrm{D}=$ data OFDM symbol

Fig. 1. Downlink MIMO MC-CDMA system with ICE

Note that $\underline{\check{S}}_{l}^{(i), m}$ has been computed using hard decision decoding.

2) Calculate the LEs $\check{H}_{l}^{(i), m, p} \forall\{l\} \in \mathcal{D}$ according to (6).

3) Obtain the final estimate of the channel transfer function between transmit antenna $m$ and receive antenna $p$ through filtering the LEs over the set $\mathcal{P}_{\text {ICE }}$ of all reference symbols, i.e.,

$$
\begin{aligned}
& \hat{H}_{n, l}^{(i), m, p}=\sum_{\left\{n^{\prime}, l^{\prime}\right\} \in \mathcal{T}_{n, l}} \omega_{n^{\prime}, l^{\prime}}^{(i)} \check{H}_{n^{\prime}, l^{\prime}}^{(i), m, p}, \\
& \mathcal{T}_{n, l} \subseteq \mathcal{P}_{\mathrm{ICE}}, \quad \forall\{n, l\} \in \mathcal{D},
\end{aligned}
$$

where $\omega_{n^{\prime}, l^{\prime}}^{(i)}$ is the Wiener filter coefficient obtained for the $i$ th iteration.

4) Use the newly estimated CSI $\hat{H}_{l}^{(i), m, p}$ from (7) in the subsequent space-time decoder and MUD/SUD block to obtain a new estimate of the transmitted information bits.

\section{Simulations}

This section presents simulation results for a downlink MIMO MC-CDMA system applying WH spreading and the Alamouti STBC with two transmit and two receive antennas and perfect $\mathrm{CE}$ (PCE), i.e., perfect channel knowledge at the receiver, PACE, and ICE.

\section{A. Simulation Parameters}

At a carrier frequency of $f_{\mathrm{c}}=5.2 \mathrm{GHz}$, the MC-CDMA systems transmits 32 OFDM symbols per frame (Figure 1(c)) divided into 768 useful data subcarriers over a bandwidth of $46.2 \mathrm{MHz}$ resulting in a subcarrier spacing of $\Delta f=60 \mathrm{kHz}$. The size of the fast Fourier transforms in the OFDM modulation and demodulation is 1024 . Thus, the sampling duration $T_{\mathrm{spl}}=1 / 61.44 \mathrm{MHz}=16.276 \mathrm{~ns}$. The guard interval $T_{\mathrm{GI}}$ is set to $256 T_{\mathrm{spl}}$. The system uses a $R=1 / 2$ convolutional mother code with generator polynomials $(561,753)_{8}$, 4-QAM symbols with Gray mapping, and WH spreading codes of length $L=32$. In the case of 64-QAM modulation, the mother code is punctured to a rate $R=3 / 4$. Except for the single-user bound (maximum-ratio combining (MRC), 1 user), $K=32$ users transmit in parallel $M=24$ data symbols per OFDM symbol that are not frequency interleaved. Six pilot symbols are inserted into the frame as in Figure 1(c) clustering two consecutive pilot symbols and spacing the clusters 12 data symbols apart. So out of the total 32 OFDM symbols in a frame 24 symbols are dedicated to data transmission. The resulting data rate varies between $27.65 \mathrm{Mbit} / \mathrm{s}$ and $124.4 \mathrm{Mbit} / \mathrm{s}$ for 4-QAM, $R=1 / 2$ and 64-QAM, $R=3 / 4$, respectively.

In the simulations the MIMO BRAN E channel is based 
on 3GPP/SCM definition for a MIMO channel exploiting multipath angular characteristics. The model parameters are adapted to the $5.2 \mathrm{GHz}$ carrier frequency and use the BRAN E channel average power delay profile [11], which refers to a typical outdoor urban multi-path propagation. The velocity that is studied is $180 \mathrm{~km} / \mathrm{h}$, which relates to a maximum Doppler frequency $f_{D_{\max }}=867 \mathrm{~Hz}$. Note, the two antennas at the base station are spaced $10 \lambda$ apart and the two antennas at the mobile terminal $0.5 \lambda$ apart [12]. This model provides realistic PSDs and correlations in space-time-frequency based on the sum of rays approach.

Since the system proposal uses full pilot OFDM symbols, frequency filtering is omitted to obtain the final channel estimates in (4) and (7). However, a robust 1-D Wiener filter is applied with 3 filter coefficients in time direction for PACE to compute the final CSI estimates in (4) from the 3 LEs (cf. (3)). To keep the same complexity for filtering in ICE, the robust 1-D Wiener filter uses 3 consecutive localized pilot and data estimates in time direction ((3) and (6)). The CE filter's Doppler PSDs is uniformly distributed with a maximum Doppler frequency $f_{\mathrm{D}_{\text {filter }}}=867 \mathrm{~Hz}=0.014 / \Delta f$.

\section{B. Simulation Results}

In Figure 2, we compare the performance of PCE, PACE, and ICE for the single-user bound (MRC, 1 user), a fullyloaded system with 32 users and MMSE SUD, different modulation schemes, and coding rates. Dashed lines denote the single-user bound and solid lines the fully-loaded system. The single-user bound for PCE performs approximately $0.3 \mathrm{~dB}$ better than the fully loaded system with MMSE SUD in Figure 2(a). The small difference is due to the fact that spread chips are placed on adjacent subcarriers. The channel coefficients of these subcarriers are strongly correlated. Hence, the channel is not very frequency selective over these neighboring subcarriers and causes only little multiple access interference (MAI) for the fully-loaded system and MMSE SUD. In the case of PACE and ICE, the difference between single-user bound and fully-loaded system increases to $1.8 \mathrm{~dB}$ as the channel estimation error causes additional MAI.

Apparently, ICE does not show any improvement over PACE in Figure 2(a). However, when comparing the frame error rate (FER) for the same simulation scenario, we always experience a small improvement of ICE vs. PACE in Figure 2(b). Note that we use only hard decision feedback to the channel estimation and no feedback information for the STBC decoding, detection, or demodulation. The iterative receiver can only improve the performance if the channel estimates due to the additional data symbol estimates are improved. [6] demonstrates that ICE can mainly compensate interpolation errors if $E_{b} / N_{0}$ is large enough. However, in the low $E_{b} / N_{0}$ region for 4-QAM in Figure 2(a), the channel estimation error is mainly due to the noisy pilot and data estimates.

Furthermore, the hard decision feedback will cause error propagation. Taking a closer look at the error propagation, we can distinguish the following two cases. In the first case, the channel decoder can correct only few errors in the received frame for the 0th iteration of ICE due to a bad channel state. Then, error propagation will further decrease the CE performance of ICE in the subsequent iterations. Thus, the total number of bit errors and the BER increases at the decoder output in the $i$ th iteration $(i>0)$, but the FER stays constant. In the second case, the decoder can correct almost all errors in the received frame. So, very few erroneous bits will propagate back in ICE, but most of the bits will be correct. Hence, CE degrades for very few subcarriers and OFDM symbols and improves for almost all others. Next, the STBC decoder, equalizer, and demodulator will input improved estimates to the decoder, which now can correct some additional errors. This will reduce the BER and the FER or keep the FER constant. Consequently, ICE does not outperform PACE for the BER in Figure 2(a) as at low $\mathrm{E}_{\mathrm{b}} / \mathrm{N}_{0}$ more bad than good channel states occur. As the FER cannot increase for bad channel states, but can decrease even for the few good channel states, ICE always shows a small improvement compared to PACE in the FER plot (Figure 2(b)).

Considering 64-QAM with a punctured convolutional code with rate $R=3 / 4$ in Figure 2(c), we first note that the degradation due to MAI has increased from $0.3 \mathrm{~dB}$ to more than $1 \mathrm{~dB} E_{b} / N_{0}$ for PCE. The differences for PACE and ICE have increased even more. Considering only 1 active user and MRC detection, we infer from 2(c) that ICE does not converge, but degrades the BER performance with each additional iteration. In the single user case, a spread chip of the data estimates only contains $1 / 32$ of the energy of a pilot symbol. Thus, the LEs in (6) will be $15 \mathrm{~dB}$ more noisy and degrade ICE vs. PACE.

A fully-loaded system with MMSE SUD yields similar results for $E_{b} / N_{0}$ smaller than $17.5 \mathrm{~dB}$. Above that $E_{b} / N_{0}$, ICE is able to outperform PACE by at most $1 \mathrm{~dB}$. In contrast to the 4-QAM case, the channel estimation error for PACE at high $\mathrm{E}_{\mathrm{b}} / \mathrm{N}_{0}$ should be mainly dominated by the interpolation error between the LEs in time direction. Moreover, the number of feedback errors is decreasing with increasing $E_{b} / N_{0}$. If the BER of the initial iteration in ICE, i.e., the BER of PACE, is below $10^{-4}$, there are only very few feedback errors in a frame. Thus, the additional LEs from (6) will reduce the interpolation error in (7) and ICE can outperform PACE at the cost of increased complexity.

Examining the FER in 2(d), we observe that in the single user case ICE degrades compared to PACE due to the $15 \mathrm{~dB}$ more noisy LEs. However, the FER does not increase with additional iterations. For a fully-loaded system, ICE always outperforms PACE as previously explained. In contrast to 4-QAM, there exists a significant gain in $\mathrm{E}_{\mathrm{b}} / \mathrm{N}_{0}$ at a FER of $10^{-2}$ for ICE vs. PACE, which is $1.5 \mathrm{~dB}$ and $2 \mathrm{~dB}$ with 1 and 2 iterations in ICE.

\section{SUMMARY AND CONCLUSIONS}

In this paper, we present a downlink MIMO MC-CDMA system with Walsh-Hadamard spreading codes and orthogonal STBCs applying PACE and ICE at the receiver. Since the superposition of Walsh-Hadamard spread data signals can result in zero-valued chips after spreading, a method must be found that avoids noise enhancement in ICE. In ICE, hard decided estimates of the transmitted data are fed back as reference signals to the channel estimation. Orthogonal STBCs enable 


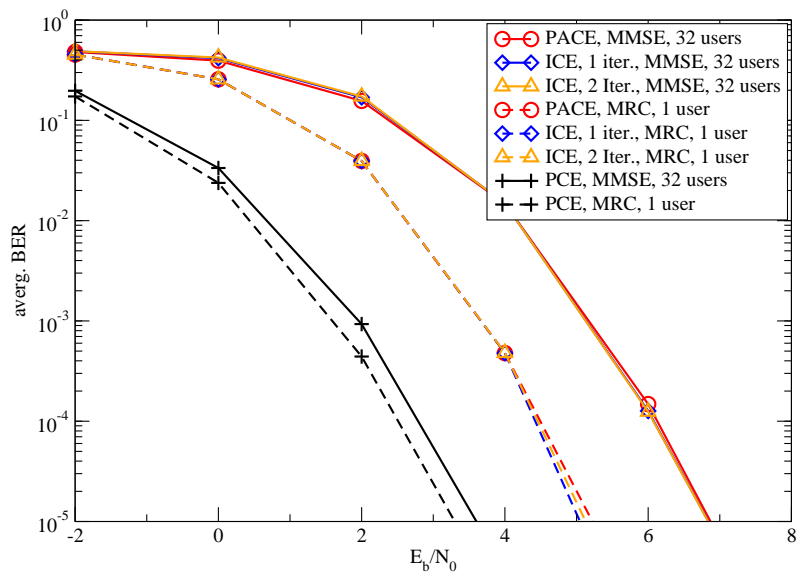

(a) BER: 4-QAM, $R=1 / 2$

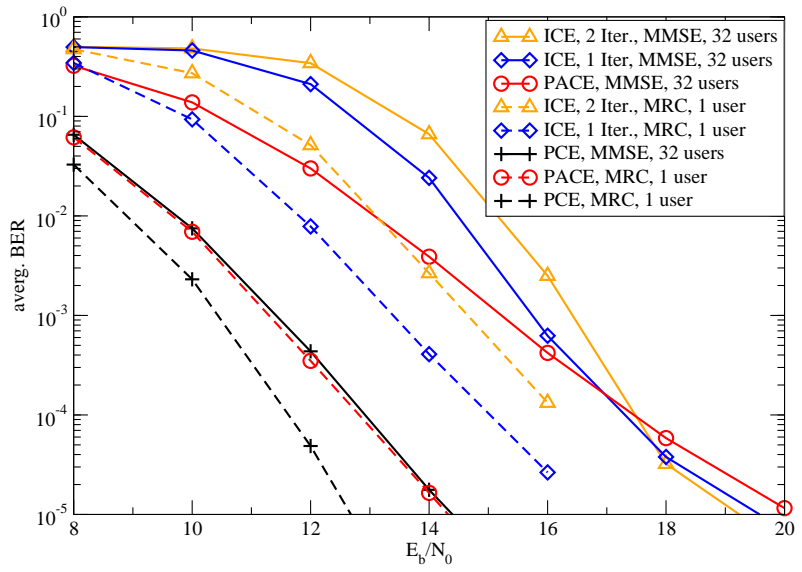

(c) BER: 64-QAM, $R=3 / 4$

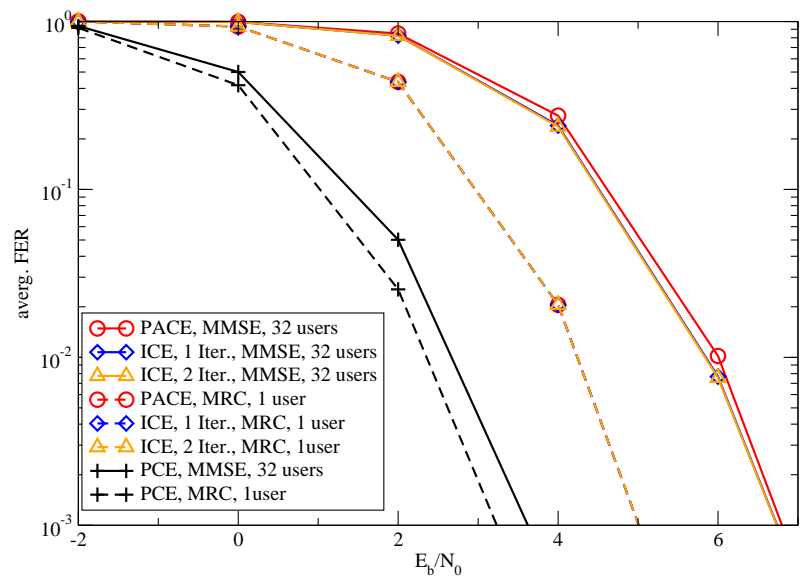

(b) FER: 4-QAM, $R=1 / 2$

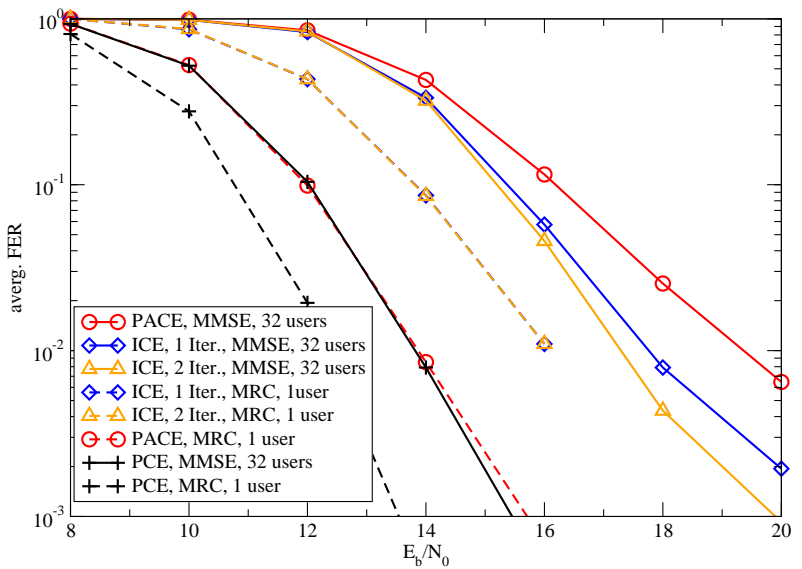

(d) FER: 64-QAM, $R=3 / 4$

Fig. 2. Comparison of perfect CE (PCE), PACE, and ICE for (a), (b) 4-QAM and $R=1 / 2$ or (c), (d) 64 -QAM $R=3 / 4$.

the computation of localized channel state information for the estimated transmit data. Thus, we propose a MIMO channel estimation method for ICE that avoids noise enhancement. Simulations were carried out using a realistic outdoor MIMO channel based on the 3GPP MIMO channel model. The results demonstrate that ICE can improve bit error rates over PACE only at high $\mathrm{E}_{\mathrm{b}} / \mathrm{N}_{0}$. However, ICE can improve PACE for small to medium $\mathrm{E}_{\mathrm{b}} / \mathrm{N}_{0}$ when comparing frame error rates.

To conclude, frame error rates seem to be a better performance measure for ICE than bit error rates. We further need to study the performance of soft decision feedback to reduce the effect of error propagation. Finally, the filtering in ICE should adaptively take the reliability of data estimates into account.

\section{ACKNOWLEDGMENT}

This research has been conducted within the European Network of Excellence NEWCOM and the European ISTProject 4MORE, which are both supported by the European Commission in the framework of FP6.

\section{REFERENCES}

[1] K. Fazel and S. Kaiser, Multi-Carrier and Spread Spectrum Systems. John Wiley and Sons, 2003.
[2] Y. Kishiyama, N. Maeda, K. Higuchi, et al., "Experiments on throughput performance above 100-Mbps in forward link for VSF-OFDCDM broadband packet wireless access," in Proc. of IEEE VTC'F03, Orlando, USA, Oct. 2003.

[3] IST MATRICE project, web-site: http://www.ist-matrice.org.

[4] IST 4MORE project, web-site: http://www.ist-4more.org.

[5] S. M. Alamouti, "A simple transmit diversity technique for wireless communications," IEEE J. Select. Areas Commun., vol. 16, no. 8, pp. 1451-1458, October 1998.

[6] F. Sanzi, S. Jelting, and J. Speidel, "A comparative study of iterative channel estimators for mobile OFDM systems," IEEE Trans. Wireless Commun., pp. 849-859, Sept. 2003.

[7] A. G. Paulo Marques, Artur Pereira, "Pilot and data aided channel estimation for uplink MC-CDMA mobile systems," in Proc. of 14th IST SUMMIT, Dresden, Germany, 2005.

[8] S. Sand, R. Raulefs, and G. Auer, "Iterative channel estimation for high mobility broadband MC-CDMA systems," in Proc. of IEEE ICC 2005, Seoul, Korea, May 2005.

[9] F. Portier, I. Raos, Silva, et al., "Transmission techniques for downlink multi-antenna MC-CDMA systems in a Beyond-3G context," $J$. of Communications and Networks, vol. 7, no. 2, pp. 157-170, Jun. 2005.

[10] 3GPP2 TR 25.996, "Network spatial channel model for multiple intput multiple output simulations (release 6)," Apr. 2003.

[11] ETSI, "Broadband Radio Access Networks (BRAN); HIPERLAN Type 2; Physical Layer," Dec. 2001.

[12] F. Portier, J.-Y. Baudais, and J.-F. Hélard, "Performance of STBC MCCDMA systems over outdoor realistic MIMO channels," in Proc. of IEEE VTC'F04, vol. 4, Los Angeles, USA, Sept. 2004, pp. 2409-2413. 